\title{
Stored Energy Predictions from Dislocation-Based Hardening Models and Hardness Measurements for Tensile-Deformed Commercial Purity Copper
}

\author{
Matjaž Čebron 1,* - Franc Kosel2 \\ ${ }^{1}$ Hidria Rotomatika, Slovenia \\ 2 University of Ljubljana, Faculty of Mechanical Engineering, Slovenia
}

\begin{abstract}
Stored energy analyses by differential scanning calorimetry (DSC) and indentation hardness measurement were performed on crosssectional samples cut from the gauge length of tensile-deformed copper specimens. The stress-strain curve was described using dislocationbased hardening models integrated into a visco-plastic Taylor-type model of polycrystal deformation. Three approaches in reproducing the experimental stress-strain curve were used to evaluate the differences in dislocation density predictions resulting from different modelling options. A good description of hardening was achieved by all three approaches and constitutive models and only negligible differences were found in the predicted dislocation density between assumed homogeneous and heterogeneous dislocation distribution throughout the polycrystal. Measured values of stored energy are somewhat lower than those published in research studies in which one-step and slow annealing methods were used. A simple model predicting a nearly linear increase of stored energy with dislocation density was found to adequately describe retained energy evolution. Since different dislocation arrangements result in different yield stress and energy predictions, both results can be used to determine values of parameters in two-internal-variable hardening models. Even though both measured quantities were satisfyingly described, uncertainties regarding material parameters and the applied polycrystal and stored energy models prevent us from claiming that the evaluated dislocation density distributions represent the actual dislocation structure in the material. As expected for strongly hardening materials, the relationship between yield stress and hardness could not be adequately approximated by a linear function. Instead, a linear combination of yield stress and hardening rate was used, finally providing a relation between hardness and stored energy through their mutual dependence on yield stress.
\end{abstract}

Keywords: dislocations, hardening models, crystal plasticity, stored energy, calorimetry, hardness

\section{INTRODUCTION}

In the last decades considerable effort has been dedicated to the development of hardening models based on the underlying physical mechanisms involved in plastic deformation of crystalline materials. Due to the complexity of the processes involved, most physically based models to date combine the theoretical knowledge of deformational behaviour with a series of empirical rules obtained from experimental observations. The plastic response of metals is determined by the production and migration of defects in the crystal lattice called dislocations [1]. Despite suggestions that a complete description of work hardening in terms of dislocation theory may never be possible, in-depth research on pure metals and heterogeneous solids has clarified many aspects of the complex processes involved [2] and [3].

Dislocation-based models are expected to have a wider applicability and better predictability than phenomenological formulations over larger ranges of strain, strain rate and temperature. Since they are based on the description of microstructural evolution, they can be handily combined with models of other structure-dependent processes affecting physical properties of the material like recristalization and recovery, e.g. in [4] to [6]. Earlier dislocation-based models of strain hardening, see [7] to [9], were mainly concentrating on explaining stage III of the hardening process and were able to describe a gradual decrease of the hardening rate, which in many cases is a nearly linear function of yield stress. This type of strain hardening behaviour can be explained in terms of constitutive models using a single internal variable related to the mean dislocation density [10]. Under deformation conditions in which plastic instabilities are avoided, the gradual decrease of the hardening coefficient is interrupted by a new hardening stage characterized by a nearly constant hardening rate which is eventually followed by a final drop-off leading to stress saturation [3]. Moreover, stored dislocations in crystalline materials are rarely distributed homogeneously throughout the structure, implying that a satisfactory description of the mechanical state in terms of the mean dislocation density is inadequate. To overcome this inconsistency and correctly describe the hardening behaviour following phase III, two-internal-variable models had to be invoked [11].

Estrin et al. [10] presented a dislocation-densitybased model aimed at describing the hardening 
behaviour of cell-forming crystalline materials studying also the effect of texture variation on strain hardening. Argon and Haasen [3] proposed a mechanism of work hardening related to a cellular dislocation microstructure in which hardening of cell interiors occurs through the build-up of long range internal stresses associated with lattice misorientations between dislocation cells. In [12] Ma and Roters presented a constitutive model for fcc crystals which follows individual slip-system hardening and can therefore directly account for latent hardening. A model for heterogeneous alloys was developed in [13] with the density of mobile dislocations added as an additional internal variable. Recently, several dislocation models focused on understanding the dislocation mechanisms accompanying stress reversal have been proposed in an effort of explaining the material response following deformation path changes, e.g. in [14] and [15]. In the presence of a heterogeneous dislocation distribution, long range internal stresses are an unavoidable consequence of compatibility requirements during deformation in the stress-applied state [3]. Experiments by Mughrabi [11] and Mughrabi et al. [16] have shown that in the loaded state both cell walls and cell interiors are subjected to stresses in the same direction as the applied stress, however stresses in walls are far larger than the applied ones while those in cell interiors are smaller. Essman [17] and [18] used fast neutron irradiation to pin dislocations of copper single crystals strained into hardening stages I and II. While he found no evidence of long range stresses in stage I the curvature of dislocations in the unstressed crystals strained into stage II allowed him to estimate long range internal stresses of the order of half the forward yield stress.

Dislocation-based hardening models provide evolutionary equations for dislocation densities and their relation to the shear strength of single crystals. In order to relate the latter to the macroscopic values of stress and strain measured on a polycrystal aggregate, the model has to be complemented by a description of polycrystal deformation. In this work a visco-plastic Taylor-type model is used. In recent years several variants of Taylor-type models have been introduced with the common quality of allowing for the active slip systems, slip rates, rates of lattice rotation and the deviatoric stress to be calculated for each grain separately. As a result, calculation time is much smaller compared to crystal plasticity FE methods or self-consistent models [19] and [20] in which grains are treated as inclusions within a homogenized medium having the average constitutive behaviour of the entire aggregate.
During plastic deformation of metals, most of the mechanical energy expended is dispersed as heat. The remaining part, often referred to as stored energy, is retained in the metal as the energy of the elastic field of the dislocation structure. The energy storage phenomenon in metals was discovered by Taylor and Quinney [21]. The retained energy can be measured by one or two-step methods. In onestep techniques the measurements take place directly during the deformation process. These methods are usually regarded as more accurate, however the need for special equipment and measuring procedures employed has favoured the use of two-step techniques with a shorter processing time and substantially lower costs [22]. In two-step methods the already deformed material is subjected to a protocol of heat treatment during which the heat flow caused by thermal processes in the specimen is measured.

It has been proposed in [23] and [24] that indentation hardness can provide a simple method for determining stored energy, since it is a local mechanical property which depends on yield stress and therefore on the local dislocation density. In this work the relationship between stored energy, yield stress and Vickers hardness for tensile-deformed copper has been studied and compared with previously published results and models.

\section{CONSTITUTIVE MODELS}

\subsection{Bergström Hardening Model}

In 1970 Bergström [8] presented a dislocation-based constitutive model aimed at describing the hardening behaviour of polycrystalline $\alpha-\mathrm{Fe}$ at room temperature. The model distinguishes between three components of yield stress:

$$
\sigma=\sigma_{0}(T)+\sigma^{*}\left(\dot{\varepsilon}^{p}, T\right)+\sigma_{d}(\rho, T) .
$$

In Eq. (1) $\sigma_{0}, \sigma^{*}$ and $\sigma_{d}$ are the lattice resistance, the strain-rate dependent temperature stress and the dislocation-density dependent hardening component, respectively. The contribution of $\sigma^{*}$ is usually small for fcc structured metals and is often neglected. Therefore the effect of temperature and strain rate on flow stress is usually introduced indirectly by describing their influence on the hardening rate affecting $\sigma_{d}$ [25]. The hardening component $\sigma_{d}$ is related to the mean dislocation density $\rho$ by the Taylor relationship:

$$
\sigma_{d}=\alpha^{\prime} G b \sqrt{\rho},
$$


where $\alpha^{\prime}$ is a constant describing the average interactions between dislocations, $G$ is the shear modulus and $b$ is the magnitude of the dislocation Burgers vector. Eq. (2) can be derived from a series of different interaction mechanisms (e.g. cutting of forest dislocations) and is widely used in dislocationdensity-based models as well as in more sophisticated single-crystal constitutive relations [26].

The density of mobile dislocations is considered to be constant while the variation of the total density is determined by four envisioned dislocation mechanisms: immobilization, re-mobilization, annihilation and creation, from which an evolutionary equation relating dislocation density to tensile strain $\varepsilon^{p}$ is deduced as:

$$
d \rho / d \varepsilon^{p}=U^{\prime}\left(\varepsilon^{p}\right)-A^{\prime}-\Omega^{\prime} \rho,
$$

where $U^{\prime}$ is a measure of the rate at which mobile dislocations are immobilized or annihilated and is inversely proportional to the dislocations mean free path, $\Omega^{\prime}$ expresses the probability of remobilization and annihilation through reactions between mobile and immobile dislocations and $A^{\prime}$ gives the rate of density variation from strain-invariant sources (e.g. grain boundaries and free surfaces).

Since plastic deformation of metals occurs by irreversible shear in discrete slip systems, a more commonly used approach is to relate the dislocation density to the total shear strain of metal grains. In this case Eqs. (1) to (3) must be rewritten in terms of shear quantities. Neglecting $\sigma^{*}$ in Eq. (1), we obtain:

$$
\begin{aligned}
& \tau=\tau_{0}+\tau_{d}=\tau_{0}+\alpha G b \sqrt{\rho}, \\
& d \rho / d \gamma=U(\gamma)-A-\Omega \rho,
\end{aligned}
$$

where $\tau$ and $\gamma$ are the shear strength of active slip systems and the total shear deformation of the crystal, respectively. Since we only consider the total shear deformation, equal and concurrent hardening of all slip systems is implied. In the field of crystal plasticity this kind of hardening is often termed isotropic [10]. In order to integrate Eq. (5), the function $U(\gamma)$ must be specified. In the original study [8] it was considered to be constant and the relation between strain and dislocation density was supposed to be nearly linear. This was justified by observing that a cellular dislocation structure consisting of hard dislocation walls and soft cell interiors forms in the very early stages of deformation and that the cell diameter $d$ rapidly attains a constant value which does not appreciably change on further straining. As the mean free path $z$ is considered to be proportional to the cell diameter it follows that $U$ has a constant value. Under these assumptions an analytical expression linking strain and dislocation density can be deduced. However, other investigations, e.g. in [26] and [27], have shown that typically in fcc structured metals the cell size $d$ decreases with density according to:

$$
d=K / \sqrt{\rho},
$$

where $K$ is a dimensionless constant ranging approx. from 10 to 20 for $\mathrm{Cu}$ [26]. Assuming that the dislocation mean free path is proportional to the cell size, $U$ can be written as:

$$
U=U_{0} \sqrt{\rho}
$$

where $U_{0}$ is a constant. Since $1 / \rho^{0.5}$ can be interpreted as the mean distance between dislocations, Eq. (6) is often seen as a manifestation of the so called principle of similitude, which states that dislocation structures refine themselves during straining in a self similar way thus retaining all ratios between different dimensions of the dislocation arrangement. The physical origin of what is called similitude is not yet understood and the related coefficient is not predictable from dislocation theory [26].

\subsection{Estrin Hardening Model}

The majority of dislocation-based models developed for describing hardening after phase III consider the material as a two-phase composite consisting of cell walls with a high dislocation density and dislocation-poor cell interiors. Different evolutionary equations for dislocation densities in the two phases give rise to separate stages of hardening. In most of these models the shear strength of individual phases is determined by the local dislocation density. In [3] Argon and Hassen presented a comprehensive elastic analysis explaining the role of specific dislocation arrangements and their resulting long-range stresses. Since these stresses are in self-equilibrium, they do not affect the yield strength of the material, however, they are fundamental for the two phases to deform in a coherent way. If such a deformation is supposed, the total shear strength is obtained by an expression resembling the so called rule of mixtures:

$$
\tau_{d}=f \tau_{w}+(1-f) \tau_{c}
$$

where $\tau_{w}, \tau_{c}$ and $f$ are the shear strength of cell walls, the shear strength of cell interiors and the volume fraction of cell walls, respectively. In [10] Estrin et 
al. presented a 2D model for describing hardening of cell-forming metal crystals at large strains. The model was later generalized for the 3D case and arbitrary strain paths in [28]. One of the main assumptions of the model states that dislocation generation only occurs in walls, while annihilation takes place both in walls and cell interiors. The density in cell interiors $\rho_{c}$ is affected by three processes, namely the creation of dislocations in walls, the loss of dislocations due to them becoming part of the wall structure and the mutual annihilation of dislocations, resulting in the following evolutionary equation:

$$
\frac{d \rho_{c}}{d \gamma}=\frac{\alpha^{*} \sqrt{\rho_{w}}}{\sqrt{3} b}-\frac{6 \beta^{*}}{b d(1-f)^{1 / 3}}-k_{0} \rho_{c},
$$

where $\alpha^{*}$ is the fraction of dislocation sources operated by dislocations coming from walls, $\beta^{*}$ is the fraction of interior dislocations becoming part of the wall structure and $k_{0}$ is a measure of the rate of annihilation of dislocations within the specific phase. Dislocation density in cell walls $\rho_{w}$ is increased by dislocations coming from cell interiors as well as the activation of dislocation sources by interior dislocations and again reduced by mutual annihilation:

$$
\frac{d \rho_{w}}{d \gamma}=\frac{6 \beta^{*}(1-f)^{2 / 3}}{b d f}+\frac{\sqrt{3} \beta^{*} \sqrt{\rho_{w}}}{f b(1-f)^{-1}}-k_{0} \rho_{w} .
$$

The specific numeric values appearing in Eqs. (9) and (10) are a result of assuming a cubic shape of cells with side length $d$. Mechanisms leading to the development of cellular dislocation patterns are not considered in the model. Shear strengths of the two phases are related to the dislocation densities therein according to the same Taylor relationship, Eqs. (2) and (4), as is used in the Bergström model. The constitutive description is completed by a scaling relation for the average cell size and a description of the evolution of the wall volume fraction. The average cell size is defined by Eq. (6) using the average dislocation density $\rho_{a}$ as the scaling measure:

$$
d=K / \sqrt{\rho_{a}}=K / \sqrt{f \rho_{w}+(1-f) \rho_{c}} .
$$

The principle of similitude implies a constant volume fraction of cell walls. However, this assumption is not supported by experimental observations showing that after an initial increase $f$ monotonically decreases to a saturation value at larger strains [10]. The variation of $f$ is described by:

$$
f=f_{\infty}+\left(f_{0}-f_{\infty}\right) \exp \left(-\gamma / \gamma_{e}\right)
$$

where $f_{\infty}$ and $f_{0}$ are the saturated and initial values and $\gamma_{e}$ is a constant describing the rate of decrease of $f$.

\subsection{Polycrystal Model}

The hardening models presented above describe the relationship between the shear strength of slip systems and the accumulated shear deformation of individual crystals. In order to relate these to the macroscopic values of deformation and stress, a model of polycrystal deformation has to be used. The following description of kinematic behaviour of crystals subjected to large deformations is based on the assumption of multiplicative decomposition of the total deformation gradient into an elastic and plastic part first proposed by Lee [29]. Three configurations of the material are introduced: initial, intermediate and current, see Fig. 1.

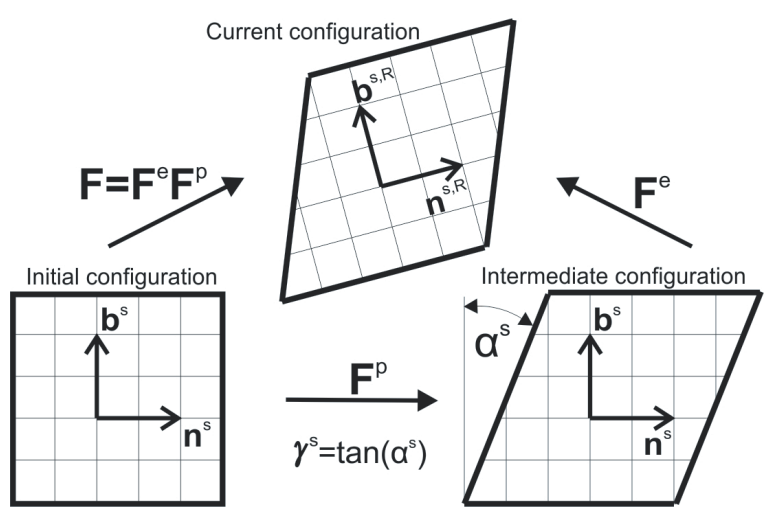

Fig. 1. Decomposition of deformation

The deformation is characterized by the velocity gradient tensor $\mathbf{L}$ and the total deformation gradient $\mathbf{F}$ describing the deformation of the material from the initial to the current configuration:

$$
F_{i j}=\partial x_{i} / \partial X_{j}, \quad L_{i j}=\partial \dot{x}_{i} / \partial x_{j},
$$

where $\mathbf{x}(\mathbf{X})$ and $\mathbf{X}$ are the current and initial coordinates of the material particle, respectively. From Eq. (13) an expression for the time derivative of the deformation gradient can be deduced:

$$
\dot{\mathbf{F}}=\frac{\partial \mathbf{v}}{\partial \mathbf{X}}=\left(\frac{\partial \mathbf{v}}{\partial \mathbf{x}}\right)\left(\frac{\partial \mathbf{x}}{\partial \mathbf{X}}\right)=\mathbf{L F} .
$$

Plastic deformation is a result of dislocation slip occurring on favourable crystallographic planes and in specified directions, together forming a slip system. 
A pair of orthogonal vectors $\left\{\mathbf{b}^{s}, \mathbf{n}^{s}\right\}$, where $\mathbf{b}^{s}$ is a unit vector parallel to the slip direction and $\mathbf{n}^{s}$ is a unit vector normal to the slip plane is used to describe slip system $s$.

The total deformation gradient $\mathbf{F}$ is decomposed into an elastic $\mathbf{F} e$ and a plastic part $\mathbf{F} p$. It is supposed that during plastic deformation the material flows through the invariant lattice while in the following elastic part the lattice deforms attached to the material. The elastic deformation gradient comprises eventual elastic deformations and rigid body rotations. Since our study is focused only on the rigid-plastic model of single crystal kinematics, no "real" elastic deformations are considered. The elastic part of the total deformation gradient thus reduces to a rigid rotation $\mathbf{R}$ :

$$
\mathbf{F}=\mathbf{F}^{e} \mathbf{F}^{p}=\mathbf{R F}^{p} .
$$

For the plastic deformation in the initial configuration we can write a relation similar to Eq. (14):

$$
\dot{\mathbf{F}}^{p}=\mathbf{L}^{p} \mathbf{F}^{p} .
$$

The plastic velocity gradient $\mathbf{L}^{p}$ is given by a linear superposition of shear rates on all slip systems:

$$
\mathbf{L}^{p}=\sum_{s} \dot{\gamma}^{s} b_{i}^{s} n_{j}^{s}
$$

where $\dot{\gamma}^{s}$ is the shear rate of system $s$. The velocity gradient $\mathbf{L}^{p}$ can be decomposed into a symmetric strain rate $\mathbf{D}^{p}$ and a skew-symmetric rotation rate $\mathbf{W}^{p}$, also known as spin:

$$
\mathbf{L}^{p}=\mathbf{D}^{p}+\mathbf{W}^{p} .
$$

Decomposing the dyadic product $\mathbf{b}^{s \otimes} \mathbf{n}^{s}$ into a symmetric part $m_{i j}^{s}$ and a skew-symmetric part $q_{i j}^{s}$ allows us to write the strain and rotation rate in terms of shear rates on active slip systems:

$$
\begin{aligned}
& \mathbf{D}^{p}=\sum_{s} \dot{\gamma}^{s} m_{i j}^{s}, \\
& \mathbf{W}^{p}=\sum_{s} \dot{\gamma}^{s} q_{i j}^{s} .
\end{aligned}
$$

By inserting Eq. (15) into Eq. (14), the total velocity gradient can be written as:

$$
\mathbf{L}=\dot{\mathbf{F}} \mathbf{F}^{-1}=\dot{\mathbf{R}} \mathbf{R}^{T}+\mathbf{R} \mathbf{L}^{p} \mathbf{R}^{T} .
$$

Similarly to Eq. (16) the total velocity gradient can be additively decomposed into a strain and a rotation rate $(\mathbf{L}=\mathbf{D}+\mathbf{W})$. Since $\mathbf{R} \dot{\mathbf{R}}^{T}$ is a skewsymmetric matrix, we obtain:

$$
\begin{gathered}
\mathbf{D}=\mathbf{R} \mathbf{D}^{p} \mathbf{R}^{T}=\mathbf{D}^{p, R}, \\
\mathbf{W}=\dot{\mathbf{R}} \mathbf{R}^{T}+\mathbf{R} \mathbf{W}^{p} \mathbf{R}^{T}=\dot{\mathbf{R}} \mathbf{R}^{T}+\mathbf{W}^{p, R} .
\end{gathered}
$$

The strain rate $\mathbf{D}$ is a simple transformation of $\mathbf{D}^{p}$ from initial into current configuration, however the rotation rate $\mathbf{W}$ contains an additional contribution. The polycrystal model must provide a value for the velocity gradient in crystals. In Taylor-type models $\mathbf{L}$ for each of the constituent grains is considered to be equal to the imposed macroscopic velocity gradient $\mathbf{L}^{*}$. Using Eqs. (17) and (20) the strain rate $\mathbf{D}^{*}$ can be written as:

$$
\mathbf{D}^{*}=\mathbf{D}=\sum_{s} \dot{\gamma}^{s} m_{i j}^{s, R}
$$

In prescribing the macroscopic strain rate $\mathbf{D}^{*}$ it is assumed that the volume does not change $\left(D_{i i}^{*}=0\right)$. The sum of right-hand sides of Eq. (22) for $i j=11,22$, 33 is also zero because of the orthogonality between the rotated vectors $\mathbf{b}$ and $\mathbf{n}$. As a result only five of the six Eqs. (22) are independent. The fcc crystal structure of copper has 12 potentially active slip systems of the $\{111\}<110>$ type. Since there are more unknown slip rates than equations prescribing the deformation, the solution of Eq. (22) is not unique. In his original work Taylor assumed that out of the possible combinations of five active slip systems the one with the minimal rate of internally dissipated frictional work will be active. This assumption however still does not lead to a unique solution for strain rates [19]. To overcome this limitation of models based on a rate insensitive idealization of slip, Asaro and Needleman [30] proposed a simple rate-dependent model in which the shear rate $\dot{\gamma}^{s}$ is uniquely defined by:

$$
\dot{\gamma}^{s}=\dot{\gamma}_{0}\left|\tau^{s} / \tau\right|^{1 / n} \operatorname{sign}\left(\tau^{s}\right)
$$

where $\tau^{s}$ is the shear stress in slip system $s, \tau$ is the shear strength of the system described by the hardening models, $\dot{\gamma}_{0}$ is a reference shear rate and the parameter $n$ characterizes the material rate sensitivity. If $n$ approaches zero, a substantial amount of slip only occurs in systems where the shear stress reaches values near the shear strength. The rate-independent response of the material is therefore retrieved as $n \rightarrow 0$. In practice it is found that for values of 0.03 or lower, the stresses obtained from Eq. (23) differ only slightly from those obtained from the rate-insensitive Taylor-Bishop theory [30]. Using Eqs. (17) and (23), the deviatoric part of the prescribed strain rate can be written as: 


$$
D_{i j}^{*}=\dot{\gamma}_{0} \sum_{s} m_{i j}^{s, R}\left|m_{k l}^{s, R} \sigma_{k l}^{\prime} / \tau\right|^{1 / n} \operatorname{sign}\left(m_{k l}^{s, R} \sigma_{k l}^{\prime}\right),
$$

where $\sigma_{k l}^{\prime}$ is the deviatoric part of the Cauchy stress tensor. Given a prescribed strain rate $\mathbf{D}^{*}$ Eq. (24) represents a system of five independent nonlinear equations for five independent deviatoric components of stress. In our model the Newton-Raphson method was used in order to solve the system of stresses. It should be emphasized that in this approach all slip systems are considered to be active. Once the slip rates have been calculated, the spin of the crystal lattice is determined from Eqs. (18) and (21):

$$
\dot{\mathbf{R}} \mathbf{R}^{T}=\mathbf{W}^{*}-\sum_{s} \dot{\gamma}^{s} q_{i j}^{s, R} .
$$

The last expression is used to update the orientation of the crystal. A detailed description of evaluating the incremental rotation matrix using Eq. (25) and the Rodrigues formulation for finite rotations employed in our model can be found in [31]. The resulting rotation is used to calculate the new direction of vectors $\mathbf{b}$ and $\mathbf{n}$ from which follows the crystallographic texture evolution.

Using the polycrystal model, the increment of shear deformation of the $i^{\text {th }}$ crystal $d \gamma_{i}$ and the shear strength of the crystal's slip systems $\tau_{i}$ can be related to the imposed deformation increment $d \varepsilon^{p}$ and the tensile stress acting on the crystal $\sigma_{i}$ via the Taylor factor $M$ :

$$
d \gamma_{i}=\sum_{s} d \gamma_{i}^{s}=M_{i}\left(\gamma_{i}\right) d \varepsilon^{p}, \quad \sigma_{i}=M_{i}\left(\gamma_{i}\right) \tau_{i}
$$

Finally two approaches can be taken to express the macroscopic value of tensile stress. In most cases, due to smaller computational costs, all the constituent crystals are considered to harden equally in relation to the average deformation of the polycrystal aggregate. An average Taylor factor $\bar{M}(\gamma)$ is used to relate the average shear deformation increment of the constituent grains $d \gamma^{r}$ to the macroscopic deformation increment:

$$
d \gamma^{r}=(1 / N) \sum_{i=1}^{N} M_{i} d \varepsilon^{p}=\bar{M} d \varepsilon^{p},
$$

where $N$ is the number of grains included in the aggregate. Similarly the average Taylor factor is then used to link the macroscopic value of tensile stress to the average shear strength $\tau^{r}\left(\gamma^{r}\right)$ described by the hardening models, which in this case is supposed to be equal for all grains:

$$
\sigma=\bar{M} \tau^{r}
$$

The second approach is to use the hardening models as constitutive equations directly describing the hardening of single crystals. The total stress is then computed as the average stress contribution from all grains in the polycrystal:

$$
\sigma=(1 / N) \sum_{i=1}^{N} M_{i} \tau_{i}
$$

In the first approach, a homogeneous distribution of dislocation density throughout all the grains of the polycrystal aggregate is supposed, with each grain hardening simultaneously to the same extent. In the second case, each grain is considered to harden according to the same hardening law but to a different extent due to the different total shear deformation imposed. Therefore a heterogeneous dislocation distribution is assumed with different dislocation densities in each of the constituent grains.

\section{EXPERIMENTAL METHODS}

Commercial rods made of $99.98 \%$ purity copper supplied by $\mathrm{AlCu}$ d.o.o. were machined to a cylindrical shape of gauge length $35 \mathrm{~mm}$ and diameter $7 \mathrm{~mm}$ according to standard ISO 6892-1 to get proportional test pieces, see Fig. 2. After machining the specimens were annealed in a protective atmosphere at $500{ }^{\circ} \mathrm{C}$ for 30 minutes. The tensile tests were carried out at $24{ }^{\circ} \mathrm{C}$ using a Zwick/Roell Z050 testing machine at a constant deformation rate of $0.5 \mathrm{~mm} / \mathrm{min}$.

The specimens were subjected to various tensile forces in the plastic region up to fracture-point with two specimens loaded to each pre-set force value. As the differences between the force-displacement curves were found to be negligible, samples deformed to the same tensile force were assumed to be subjected to the same tensile stress.

Cross-sectional samples for DSC $(2 \times)$ and hardness measurements were obtained from each deformed specimen by cutting circular discs with a thickness of 1 to $1.5 \mathrm{~mm}$ (calorimetry) and 5 to $6 \mathrm{~mm}$ (hardness) from the gauge length perpendicular to the tensile axis. Two cutting methods were used in order to estimate possible effects of the cutting process on stored energy and hardness results. One sample from each specimen was first cut using a wire ED machine. The second group of samples was obtained using a Struers Secotom-15 cut-off machine at a cutting speed of approx. $42 \mathrm{~m} / \mathrm{s}$ and a feed rate of $0.3 \mathrm{~mm} / \mathrm{min}$. Since ED machining causes a local increase of temperature and mechanical cutting induces further hardening in the surface layers, an opposite effect on hardness and stored energy was expected from the two cutting 
processes. However, no appreciable differences in either of the measured quantities for differently cut samples were found, from which it was concluded that neither of the cutting methods had a noticeable effect on the measured results.

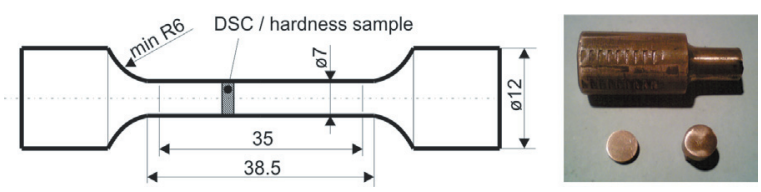

Fig. 2. Dimensions of tensile test specimens (in $\mathrm{mm}$ )

Stored energy measurements were performed using a Mettler Toledo TGA/DSC1 machine incorporating a heat-flux type differential scanning calorimeter. Alumina crucibles were used and the DSC was purged by nitrogen gas with a flow of $50 \mathrm{ml} /$ min. Samples were heated from room temperature to $500{ }^{\circ} \mathrm{C}$ at a constant rate of $10{ }^{\circ} \mathrm{C} / \mathrm{min}$. The weight of the samples was between 250 to $450 \mathrm{mg}$.

Hardness measurements were carried out using an Emcotest Durascan 20 testing machine with a standard Vickers $136^{\circ}$ pyramidal diamond indenter. All the measurements were made at room temperature; the load was set to $5 \mathrm{~kg}$ and applied for a standard dwell time of $15 \mathrm{~s}$.

\section{RESULTS AND DISCUSSION}

A random distribution of 1000 grain orientations was generated representing an initially isotropic polycrystal aggregate. Three approaches for reproducing the experimental stress-strain curve were used in order to evaluate the differences in dislocationdensity predictions resulting simply from different modelling options. In approach A Eqs. (27) and (28) were employed and the texture development was neglected, therefore the Taylor factors were constant and equal to their values at zero strain. The same equations with texture evolution taken into account were used in B, while in C Eq. (29) was employed with again considering texture changes.

The measured stress-strain curve of the material is presented in Fig. 3 along with the modelled curves and loading stresses of specimens. Material and model parameters used in the computations are given in Table 1 and Fig. 4. It is evident that a good description of hardening can be achieved by any of the employed modelling approaches and both hardening models. The differences between the modelled curves are negligible and cannot be seen from Fig. 3 where they seemingly overlap each other and the experimental results.

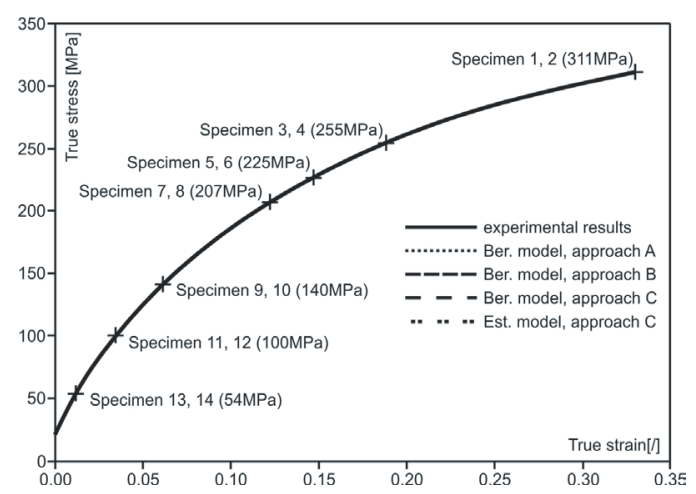

Fig. 3. Measured stress-strain curve and spec. load

Table 1. Parameters of the Bergström model

\begin{tabular}{cccc}
\hline Approach & $U_{0}\left[\mathrm{~mm}^{-1}\right]$ & $A[\mathrm{~mm}-2]$ & $\Omega[-]$ \\
\hline A & $1.059 \times 10^{5}$ & $-2.998 \times 10^{8}$ & 3.507 \\
\hline B & $1.116 \times 10^{5}$ & $-2.779 \times 10^{8}$ & 3.875 \\
\hline C & $9.414 \times 10^{4}$ & $-3.418 \times 10^{8}$ & 3.222 \\
\hline
\end{tabular}

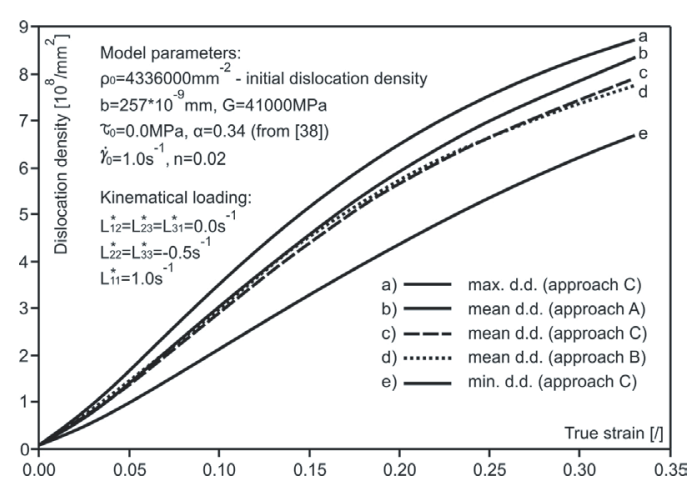

Fig. 4. Predicted dislocation density (Bergström model)

Predicted dislocation densities from the Bergström model are presented in Fig. 4. Surprisingly, the mean densities from $\mathrm{B}$ and $\mathrm{C}$ are almost identical. This results justifies using the simpler approach $B$ even though the heterogeneous distribution of dislocation density related to the actual deformation of single crystals theorized in $\mathrm{C}$ is probably betterfounded. Approach $\mathrm{C}$ also allows us to predict the range of density present in the individual grains of the polycrystal. On the other hand, using approach A results in markedly different predictions from $\mathrm{B}$ and $\mathrm{C}$ (approx. 6\% at final strain). Using constant values of Taylor factors is the most common approach for incorporating dislocation-based hardening models in continuum mechanics FE computations, e.g. in [32]. However if we are interested in dislocation density predictions, not accounting for texture evolution must lead to erroneous results [10], with approach A in our case overestimating the extent of hardening compared to $\mathrm{B}$ and $\mathrm{C}$. 
Differential calorimeters measure differences in heat flow between the test sample and the reference as a function of time or temperature. With no appreciable thermodynamic processes taking place only a slight variation of this difference can be observed. However, at the onset of a thermal event in the material, an abrupt change in the difference of heat flows occurs which is visible as a peak in the DSC curve. The area bounded by the peak and a measured or approximated baseline is equal to the material's change of enthalpy. In our study the non-isothermal heat treatment of samples produced exothermic peaks related to recrystallization. Since the pressure work and the entropy contribution due to dislocation annihilation are both negligible [22], the measured enthalpy change can be equated to the free energy present in the form of the elastic energy of the dislocation structure. Contributions from other possible strain-induced defects (e.g. point defects and twins) along with the enthalpy change due to altering grain boundaries during recrystallization are neglected. In Fig. 5 the DSC curve of one of the calorimetry samples cut from tensile specimen 3 is presented. The measured stored energies were in the range of 20 to $160 \mathrm{~mJ}(0.07$ to $0.36 \mathrm{~J} / \mathrm{g})$. Meaningful results could only be obtained from specimens 1 to 10 as the values from specimens 11 to 14 were too low to be accurately determined by the used equipment. The measured values of stored energy are somewhat lower than those published in studies where one-step and slow annealing methods were used, see [22] and [33], however comparable to other results obtained by DSC, e.g. in [23].

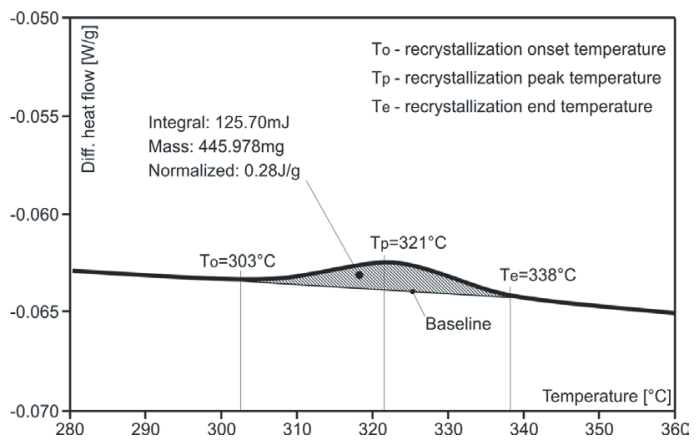

Fig. 5. DSC curve of sample taken from specimen 3

Since two-phase dislocation models imply the existence of long-range internal stresses, the energy stored in the material after unloading is not only the self energy of dislocations, but also the energy of the long-range stress field. Different measuring techniques will produce different results, since they measure different portions of this energy. Long-range stresses are often associated with the Bauschinger effect and other transient hardening phenomena observable during changes in the direction of straining [15] and [34]. It has been reported in [34] that a mild heat treatment below the recrystallization temperature tends to remove the permanent Bauschinger softening which is attributed to long-range compatibility stresses even though no appreciable changes in dislocation density can be observed at this stage [33]. It is reasonable to presume that during heating below the recrystallization point small changes in the dislocation structure occur causing long-range stresses to slowly anneal out. Although only one peak is present in the DSC curve some exothermic processes may still be proceeding during the flat phases of the curve in a slow, continuous manner therefore being undetectable by the DSC method. In one-step techniques this contribution is measured directly while in slow annealing methods it can usually only be estimated [22]. We can therefore assume that the energy measured by DSC is that of a relaxed dislocation structure with a negligible contribution from long-range stresses.

Dislocations densities resulting from approach $\mathrm{C}$ were used in stored energy evaluations. A relatively simple model for the energy of dislocations was employed in which predominantly edge-type dislocations are assumed to accumulate during the hardening process ( $v=$ Poisson`s ratio, $\rho^{m}=$ density):

$$
E_{s}=\frac{1}{N} \sum_{i=1}^{N} \frac{\rho_{i} G b^{2}}{4 \pi(1-v) \rho^{m}}\left(\ln \left(\frac{P}{\rho_{i}^{1 / 2} b}\right)+Q\right) .
$$

Eq. (30) gives the self energy of edge-type dislocations with $P / \rho_{i} 0.5$ taken as the upper and the magnitude of the Burgers vector $b$ as the lower cut-off radius, while $Q$ describes the energy of the dislocation core. Both $P$ and $Q$ are dimensionless parameters. By taking $P=1$, an alternating dipolar arrangement of positive and negative dislocations is assumed with a shielding distance of the order of the mean dislocation spacing. A similar expression can be derived by considering the total energy of a dislocation dipole in a finite cylinder, see [35], yielding only negligibly different results from Eq. (30). For $Q$ a value of 1 is used which is near the lower limit of energy usually attributed to dislocation cores [36].

Using $P=1$ as the proportionality factor between the upper cut-off radius and the mean dislocation spacing generally leads to an underestimated value of stored energy [33] and [37], which is also the case in our study, see Fig. 6 . The best fit with the measured results was obtained by taking $P=800$. This value of 
$P$ gives an unrealistically high upper cut-off radius for a random dislocation arrangement. However, it should also be acknowledged that there are two main reasons why the evaluated dislocation density is likely to be underestimated in our computations. The first is the applied polycrystal model. Taylor-type models represent an upper-bound regarding the stiffness of the polycrystal aggregate, therefore they predict the lower values of shear strength and dislocation density. The lower bound is represented by the so called Sachs-type models. The ratio of average Taylor factors between upper and lower bound models is approx. 1.37.

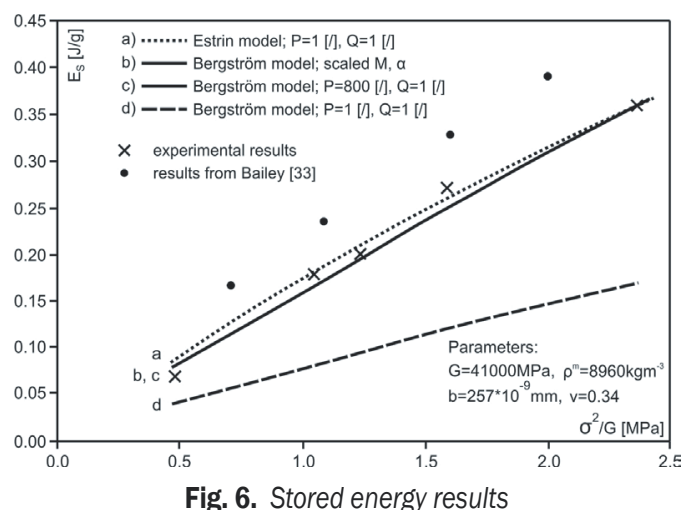

The second reason is the dependence of the strengthening coefficient $\alpha$ on dislocation density which is not described by the applied hardening models. It has been noted in [26] that dislocation densities evaluated by using constant values for $\alpha$ are probably underestimated since the line tension of dislocations declines with increasing density and the measured values of $\alpha$ usually pertain to lower concentrations in which experimental observations of dislocations are feasible. However, to the authors's knowledge, this effect has not been included in any of the dislocation-density-based hardening models presented to date. A description of the drift of the hardening coefficient is provided in [26] by Eq. (A3). By using $\alpha_{\text {ref }}=0.34$ and $\rho_{\text {ref }}=10^{7} \mathrm{~mm}^{-2}$ from [38] in Eq. (A3) [26] and scaling the Taylor factors evaluated from our polycrystal model by 0.86 , the computed dislocation density is increased enough to satisfactorily predict experimental stored energy results with $P=1$. It should be noted that scaled values of Taylor factors and strengthening coefficient used are still well within reported experimental and computational values.

In one-internal-variable models, the relation between shear strength and dislocation density is unique. On the other hand, two-internal-variable models allow for different dislocation arrangements to result in equal values of shear strength. Since different dislocation arrangements also result in different stored energy predictions, it follows that, given a known energy model, the measured values of stored energy can be used to determine the parameters of the hardening model. For evaluating the energy of the dislocation structure described by the Estrin model, Eq. (30) must be modified in order to account for the heterogeneous dislocation distribution:

$$
\begin{aligned}
E_{s} & =\frac{1}{N} \sum_{i=1}^{N}\left(\frac{\rho_{w}^{i} f_{w}^{i} G b^{2}}{4 \pi(1-v) \rho^{m}}\left(\ln \left(\frac{P}{\sqrt{\rho_{w}^{i}} b}\right)+Q\right)+\right. \\
& +\frac{1}{N} \sum_{i=1}^{N}\left(\frac{\rho_{c}^{i} f_{c}^{i} G b^{2}}{4 \pi(1-v) \rho^{m}}\left(\ln \left(\frac{P}{\sqrt{\rho_{c}^{i}} b}\right)+Q\right)\right)
\end{aligned}
$$

Material parameters presented in Tables 1 and 2 were determined from the experimental data by the least square method in combination with a simplex optimization algorithm. For the Bergström model only the stress-strain curve was modelled with the three constants in Eqs. (5) and (7) used as fitting parameters. Both, the stress-strain curve and the results of energy measurements (taking $P=Q=1$ in Eq. (31)) were used to determine material parameters of the Estrin model in Eqs. (9) to (11), while those in Eq. (12) were only allowed to vary slightly within bounds estimated from experimental results presented by Müller et al. in [39].

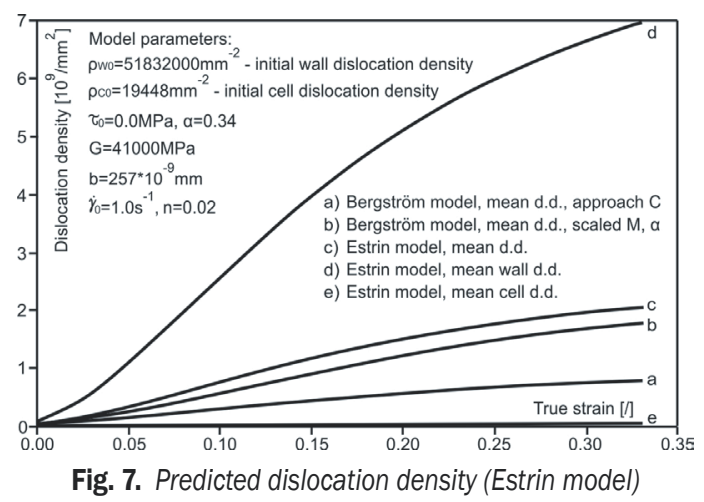

Table 2. Parameters of the Estrin model

\begin{tabular}{cccc}
\hline$\alpha^{*}[-]$ & $\beta^{*}[/]$ & $k_{0}[-]$ & $K[-]$ \\
\hline 0.027738 & 0.018175 & 4.8876 & 42.726 \\
\hline$f_{\infty}[-]$ & $f_{0}[-]$ & $\gamma_{e}[/]$ & \\
\hline 0.21678 & 0.29529 & 15.699 & \\
\hline
\end{tabular}

The mean dislocation density predicted by the Estrin model is, naturally, considerably higher than in the case of the Bergström model, see Fig. 7, since the 
calculated stored energy values were substantially too low using the same parameters in Eq. (30).

In [40] Zehetbauer and Seumer used TEM, calorimetry and electrical resistivity measurements to study the evolution of dislocation density in $99.95 \%$ purity copper deformed at room temperature. Despite the heterogeneous distribution of dislocations the shear strength was found to be proportional to the square root of the average dislocation density, perfectly in line with the one-internal-variable description of hardening. By using a constant Taylor factor of 3.06 they determined the strengthening coefficient $\alpha$ to be 0.27 . Taking into account the evolution of the average Taylor factor given by our polycrystal model the best fit is obtained with $\alpha=0.263$. This result can be implemented into the Bergström model by simply setting the value of the strengthening coefficient to 0.263 . On the other hand the parameters of the Estrin model have to be determined purposely to retrieve the same experimentally determined average density-strength relationship (and naturally reproduce the measured stress-strain curve). The coefficients used to describe the hardening of different phases in this instance need not to be equal to that determined for the average density. The predicted dislocation densities from both models (using approach C) in the aforementioned cases are presented in Fig. 8.

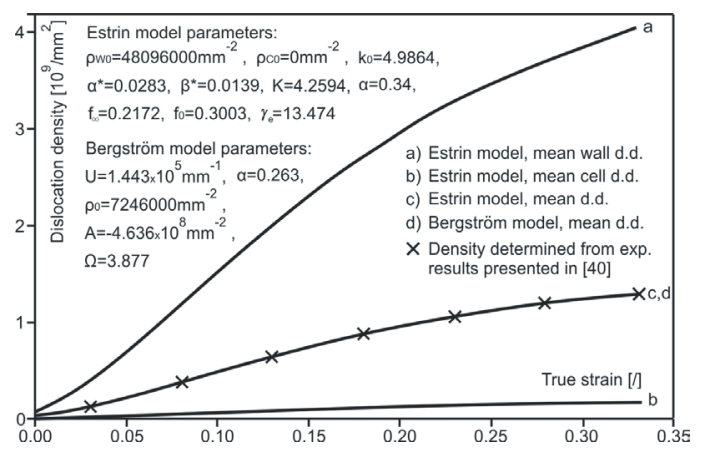

Fig. 8. Predicted dislocation densities

The best description of the measured stored energy values is achieved by taking $P=6$ for the results from the Bergström model and $P=7.3$ for the dislocation densities predicted by the Estrin model, see Fig. 9. Considering that dislocations are arranged in different slip systems these results present a very reasonable estimate of the upper cutoff radius of the order of the actual average spacing between dislocations. Naturally, using these results in the previously presented procedure of determining material parameters of the Estrin model, the same results as those already exhibited in Fig. 8 can be retrieved. Interestingly, despite the vastly diverse density distribution predicted by the two models the average energy per line length differs by only $\approx 8 \%$.
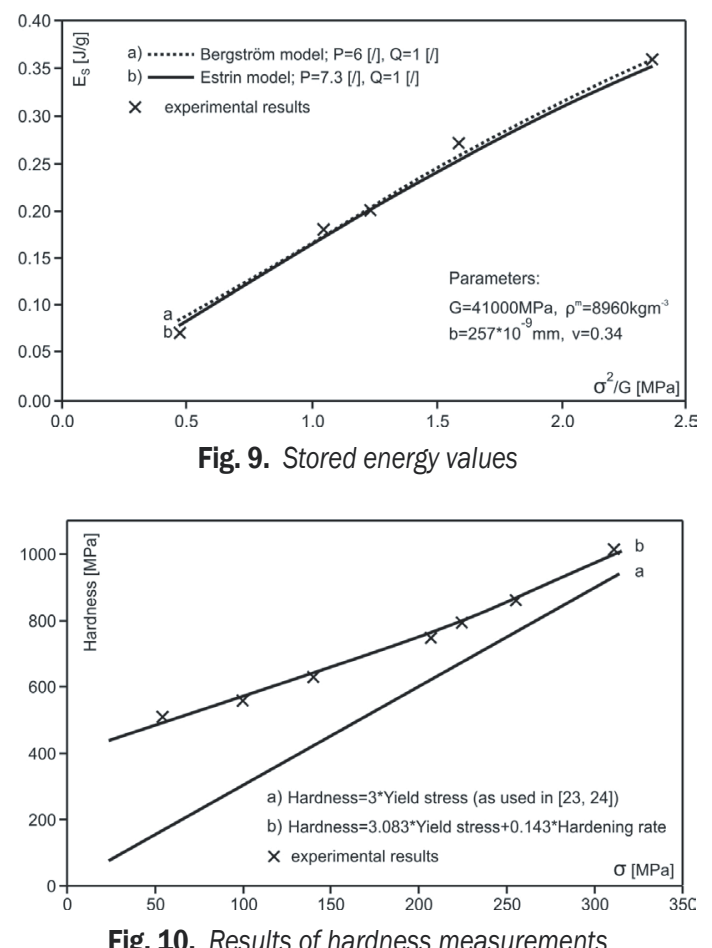

In [23] and [24] an expression predicting a linear relationship between the stored energy and the squared value of yield stress was used. It is evident from results presented in Figs. 6 and 9 that a nearly linear model also results from our study and adequately describes stored energy evolution. In the same research a linear dependence was also supposed between yield stress and hardness, which in our case is not a satisfactory approximation. Fig. 10 shows that the measured hardness values cannot be described by a linear function but can be adequately approximated by a linear combination involving both yield stress and hardening rate (which in turn can also be described as a function of yield stress). This finally provides a relation between hardness and stored energy through their mutual dependence on yield stress, which for stored energy is given by Eqs. (4), (29), and (30) in the case of the one-internal-variable Bergström model.

\section{CONCLUSIONS}

Standard specimens made from $99.98 \%$ purity copper were annealed and subjected to different tensile stresses in the plastic region of deformation. DSC and indentation hardness analyses were performed on 
cross-sectional samples cut from the gauge length of tensile specimens. The measured stress-strain curve was described using dislocation-based constitutive models. Two hardening models and their integration into a visco-plastic Taylor-type model of polycrystal deformation are presented. Three approaches for reproducing the experimental stress-strain curve were used to evaluate the differences in dislocationdensity predictions resulting from different modelling options. Unexpectedly, only negligible differences were found in the predicted mean dislocation densities between assumed homogeneous and heterogeneous density distribution through the polycrystal, while not accounting for texture evolution had a substantial influence on the results. A good description of the hardening curve was achieved by all the employed modelling approaches and both hardening models.

Values of stored energy were found to be lower than those measured in previous research studies by one-step and slow annealing methods. This probably results from the inability of the DSC method to measure energy released prior to recrystallization when during heating a slow relaxation of long range internal stresses occurs. For stored energy computations, dislocation densities from approach $\mathrm{C}$ were used. Since the part of Eq. (30) that is nonlinear in dislocation density is only logarithmic, stored energy predictions from $\mathrm{B}$ and $\mathrm{C}$, similarly to the mean dislocation density, only barely differ. A simple model predicting a nearly linear increase of stored energy with dislocation density was found to adequately describe stored energy evolution. Supposing a dipolar dislocation distribution $(P=1)$ always resulted in an underestimation of the measured results. It should be mentioned that in many studies concerning the energy of lattice defects, the self energy of dislocations is still evaluated by using $P=1$ and simply neglecting the core contribution $Q$, e.g. in [41], without any experimental validation. Besides from assuming an inadequately over-relaxed dislocation arrangement, the differences in measured and computed stored energy can also be attributed to an underestimated value of dislocation density. The scaled values of Taylor factors and strengthening coefficient used for illustrating the effect of parameter and model uncertainties on dislocation density and stored energy predictions are well within reported experimental and computational values.

Due to their good descriptive abilities dislocationbased models are becoming a popular option for describing hardening characteristics of fcc metals. Frequently, these models are only calibrated to obtain a good description of hardening, while their physical foundation in the theory of dislocations is disregarded. In these cases they can be viewed as purely phenomenological formulations. Recently a few approaches for ensuring dislocation densities and other dislocation structure parameters to stay within physically reasonable boundaries have been presented, e.g. in [42]. The use of two-internal variable models presents several difficulties regarding the determination of the exact values of material parameters. It is virtually impossible to determine the exact values of the hardening coefficients inside separate phases. Furthermore experimental studies regarding the exact density distribution are still quite scarce and hardly any general conclusions can be retrieved from them (for example the estimated values of the ratio of dislocation densities in cell interiors and cell walls vary from around 0.5 in [43] to 0 in [3]). Various researchers have questioned the use of composite type models especially since the vast majority of experimental studies have found the shear strength to be proportional to the square root of the average dislocation density regardless of the exact arrangement of dislocations, e.g. in [40]. This has also lead to the development of hybrid models in which the evolution of dislocation densities in separate phases of the cellular structure is still described by different evolutionary equations while the shear strength of the crystal is given by the Taylor relationship in terms of the average dislocation density only, e.g. in [42].

The dependence of yield stress and stored energy on dislocation structure in two-internalvariable models allows us to use the measured stress-strain curve and stored energy results to determine the parameters of the Estrin hardening model using pre-established yield stress and retained energy expressions. Even though both measured results are satisfyingly described by the model in combination with Eq. (31), it is evident from previous considerations that uncertainties regarding material parameters, the applied polycrystal model, the stored energy model and the accuracy of measurements of stored energy prevent us from claiming that the evaluated dislocation density and distribution are an accurate representation of the dislocation structure present in the material. Energy models and stored energy measurements are not accurate enough to capture the effects of smaller variations in the dislocation arrangement. Therefore they must not be regarded as an alternative to direct observations of the evolution of dislocations structures during hardening or other methods of measuring dislocation density usually used for validating dislocation-based hardening models. Their use in our research should 
instead be viewed as a quick and inexpensive way of assuring that the dislocation densities evaluated from hardening models remain within a physically acceptable range.

Finally very reasonable values of dislocation selfenergies which can be used in procedures of validation and determination of model parameters were obtained from experimentally determined results regarding dislocation density evolution presented by Zehetbauer and Seumer [40].

As expected for strongly hardening materials, the relationship between yield stress and Vickers hardness in our case could not be adequately approximated by a linear function. Instead a linear combination of yield stress and hardening rate was derived, giving a considerably better description of the measured hardness values. For slow and non-hardening materials our approximation closely resembles the relationship used in [23] and [24].

\section{ACKNOWLEDGMENTS}

This research was partly financed by the European Union (European Social Fund, Programme for Human Resources Development for the Period 2007-2013).

\section{REFERENCES}

[1] Schoek, G. (1956). Dislocation theory of plasticity of metals. Advances in Applied Mechanics, vol. 4, p. 229279, DOI:10.1016/S0065-2156(08)70374-0.

[2] Cottrel, A.H. (1985). Dislocations and Properties of Real Materials. The Institute of Metals, London.

[3] Argon, A.S., Haasen, P. (1993). A new mechanism of work hardening in the late stages of large strain plastic flow in f.c.c. and diamond cubic crystals. Acta Metallurgica et Materialia, vol. 41, no. 11, p. 32893306, DOI:10.1016/0956-7151(93)90058-Z.

[4] V.d. Boogaard, A.H, Huétink, J. (2006). Simulation of aluminium sheet forming at elevated temperatures. Computer Methods in Applied Mechanics and Engineering, vol. 195, no. 48-49, p. 6691-6709, DOI:10.1016/j.cma.2005.05.054.

[5] Lin, J., Dean, T.A. (2005). Modelling of microstructure evolution in hot forming using unified constitutive equations. Journal of Materials Processing Technology, vol. 167, no. 2-3, p. 354-362, DOI:10.1016/j. jmatprotec.2005.05.026.

[6] Marx, V., Reher, F.R., Gottstein, G. (1999). Simulation of primary recrystallization using a modified 3D cellular automaton. Acta Materialia, vol. 47, no. 4, p. 1219-1230, DOI:10.1016/S1359-6454(98)00421-2.

[7] Kocks, U.F. (1976). Laws for work-hardening and low-temperature creep. Journal of Engineering
Materials and Technology, vol. 98, no. 1, p. 76-85, DOI:10.1115/1.3443340.

[8] Bergström, Y. (1970). A dislocation model for the stress-strain behaviour of polycrystalline $\alpha$-Fe with special emphasis on the variation of the densities of mobile and immobile dislocations. Materials Science and Engineering, vol. 5, no. 4, p. 193-200, DOI:10.1016/0025-5416(70)90081-9.

[9] Mecking, H., Kocks, U.F. (1981). Kinetics of flow and strain-hardening. Acta Metallurgica, vol. 29, no.11, p. 1865-1875, DOI:10.1016/0001-6160(81)90112-7.

[10] Estrin, Y., Tóth , L.S., Molinari, A. Bréchet, Y. (1998). A dislocation-based model for all hardening stages in large strain deformation. Acta Materialia, vol. 46, no. 15, p. 5509-5522, DOI:10.1016/S1359-6454(98)001967.

[11] Mughrabi, H. (1987). A two-parameter description of heterogeneous dislocation distributions in deformed metal crystals. Materials Science and Engineering, vol. 85, p. 15-31, DOI:10.1016/0025-5416(87)90463-0.

[12] Ma, A., Roters, F. (2004). A constitutive model for fcc single crystals based on dislocation densities and its application to uniaxial compression of aluminium single crystals. Acta Materialia, vol. 52, no. 12, p. 3603-3612, DOI:10.1016/j.actamat.2004.04.012.

[13] Roters, F., Raabe, D., Gottstein, G. (2000). Work hardening in heterogeneous alloys - a microstructural approach based on three internal state variables. Acta Materialia, vol. 48, no. 17, p. 4181-4189, DOI:10.1016/ S1359-6454(00)00289-5.

[14] Barlat, F., Ferreira Duarte, J.M., Gracio J.J., Lopes, A.B., Rauch, E.F. (2003). Plastic flow for nonmonotonic loading conditions of an aluminum alloy sheet sample. International Journal of Plasticity, vol. 19 , no. 8 , p. 1215-1244, DOI:10.1016/S07496419(02)00020-7.

[15] Viatkina, E.M., Brekelmans, W.A.M., Geers, M.G.D. (2007). Modelling the evolution of dislocation structures upon stress reversal. International Journal of Solids and Structures, vol. 44, no. 18-19, p. 6030-6054, DOI:10.1016/j.ijsolstr. 2007.02.010.

[16] Mughrabi, H., Ungár, T., Kienle, W., Wilkens, M. (1986). Long-range internal stresses and asymmetric X-ray line-broadening in tensiledeformed [001]-orientated copper single crystals. Philosophical magazine A, vol. 53, no. 6, p. 793-813, DOI:10.1080/01418618608245293.

[17] Essmann, U. (1965). Elektonenmikroskopische untersuchung der versetzungsanordnung verformter kupfereinkristalle I. Die versetzungsanordnung im bereich I. Physica Status Solidi, vol. 12, no. 2, p. 707722, DOI:10.1002/pssb.19650120218.

[18] Essmann, U. (1965). Elektonenmikroskopische untersuchung der versetzungsanordnung verformter kupfereinkristalle II. Die versetzungsanordnung im bereich II. Physica Status Solidi, vol. 12, no. 2, p. 723747, DOI:10.1002/pssb.19650120219. 
[19] Van Houtte , P., Saiyi, L., Seefeld, M., Delannay, L. (2005). Deformation texture prediction: from the Taylor model to the advanced Lamel model. International Journal of Plasticity, vol. 21, no. 3, p. 589-624, DOI:10.1016/j.ijplas.2004.04.011.

[20] Molinari, A., Ahzi, S., Kouddane, R. (1997). On the self-consistent modeling of elastic-plastic behavior of polycrystals. Mechanics of Materials, vol. 26, no. 1, p. 43-62, DOI:10.1016/S0167-6636(97)00017-3.

[21] Taylor, G.I., Quinney, H. (1933). The latent energy remaining in a metal after cold working. Proceedings of the Royal Society A, vol. 143, no. 849, p. 307-326, DOI:10.1098/rspa.1934.0004.

[22] Bever, M.B., Holt, D.L., Titchener, A.L. (1973). The stored energy of cold work. Progress in Materials Science, vol. 17, p. 5-177, DOI:10.1016/00796425(73)90001-7.

[23] Kazeminezhad, M. (2008). Relation between the stored energy and indentation hardness of copper after compression test: models and measurements. Journal of Materials Science, vol. 43, no. 10, p. 3500-3504, DOI:10.1007/s10853-008-2454-z.

[24] Taheri, M., Weiland, H., Rollett, A. (2006). A method of measuring stored energy macroscopically using statistically stored dislocations in commercial purity aluminium. Metallurgical and Materials Transactions $A$, vol. 37, no.1, p. 19-25, DOI:10.1007/s11661-0060148-1.

[25] Bergström, Y., Hallén, H. (1982). An improved dislocation model for the stress-strain behaviour of polycrystalline $\alpha$-Fe. Materials Science and Engineering, vol. 55, no. 1, p. 49-61, DOI:10.1016/0025-5416(82)90083-0.

[26] Sauzay, M., Kubin, L.P. (2011). Scaling laws for dislocation microstructures in monotonic and cyclic deformation of fcc metals. Progress in Materials Science, vol. 56, no. 6, p. 725-784, DOI:10.1016/j. pmatsci.2011.01.006.

[27] Holt, D.L. (1970). Dislocation cell formation in metals. Journal of Applied Physics, vol. 41, no.8, p. 3197-3201, DOI:10.1063/1.1659399.

[28] Tóth, L.S., Molinari, A., Estrin, Y. (2002). Strain hardening at large strains as predicted by dislocation based polycrystal plasticity model. Journal of Engineering Materials and Technology, vol. 124, no. 1, p. 71-77, DOI: 10.1115/1.1421350.

[29] Lee, E. (1969). Elastic-plastic deformation at finite strains. Journal of Applied Mechanics, vol. 36, no. 1, p. 1-6, DOI:10.1115/1.3564580.

[30] Asaro, R.J., Needleman, A. (1985). Texture development and strain hardening in rate dependent polycrystals. Acta Metallurgica, vol. 33, no. 6, p. 923953, DOI:10.1016/0001-6160(85)90188-9.

[31] Tome, C.N., Lebensohn, R.A. (2012). Manual for code, Visco-plastic self-consistent (VPSC), - updated April 1, 2012. In PDF version from ftp://ftp.lanl.gov/public accessed on 23.04.2013.
[32] Hosseini, E., Kazaminezhad, M. (2011). Implementation of a constitutive model in finite element method for intense deformation. Materials and Design, vol. 32, no. 2, p. 487-494, DOI:10.1016/j. matdes.2010.08.033.

[33] Bailey, J.E. (1963). The dislocation density, flow stress and stored energy in deformed polycrystalline copper. Philosophical Magazine, vol. 8, no. 86, p. 223-236, DOI:10.1080/14786436308211120.

[34] Pedersen, O.B., Brown, L.M., Stobbs, W.M. (1981). The bauschinger effect in copper. Acta Metallurgica, vol. 29, no. 11, p. 1843-1850, DOI:10.1016/00016160(81)90110-3.

[35] Hirth, J.P., Lothe, J. (1982). Theory of Dislocations, $2^{\text {nd }}$ ed. John Wiley \& Sons, New York.

[36] Friedel, J. (1964). Dislocations. Pergamon Press, Oxford.

[37] Haessner, F., Schmidt, J. (1988). Recovery and recrystallization of different grades of high purity aluminium determined with a low temperature calorimeter. Scripta Metallurgica, vol. 22, no. 12, p. 1917-1922, DOI:10.1016/S0036-9748(88)80238-2.

[38] Gottler, E. (1973). Versetzungsstruktur und verfestigung von [100]-kupfereinkristallen, I. Verstezungsanordnung und zellstruktur zugverformter kristalle. Philosophical Magazine, vol. 28, no. 5, p. 1057-1076, DOI:10.1080/14786 437308220968.

[39] Müller, M., Zehetbauer, M., Borbély, A., Ungár, T. (1996). Stage IV work hardening in cell forming materials, part I: Features of the dislocation structure determined by X-ray line broadening. Scripta Materialia, vol. 35, no. 12, p. 1461-1466, DOI:10.1016/ S1359-6462(96)00319-3.

[40] Zehetbauer, M., Seumer, V. (1993). Cold work hardening in stages IV and V of fcc. metals - I. Experiments and interpretation. Acta Metallurgica et Materialia, vol. 41, no. 2, p. 577-588, DOI:10.1016/0956-7151(93)90088-A.

[41] Schafler, E., Steiner, G., Korznikova, E., Kerber, M., Zehetbauer, M.J. (2005). Lattice defect investigation of ECAP-Cu by means of X-ray line profile analysis, calorimetry and electrical resistometry. Materials Science and Engineering A, vol. 410-411, p. 169-173, DOI:10.1016/j.msea.2005.08.070.

[42] Silbermann, C.B., Shutov, A.V., Ihlemann, J. (2013). Modelling the evolution of dislocation populations under non-proportional loading. International Journal of Plasticity, vol. 55, p. 58-79, DOI:10.1016/j.ijplas. 2013.09.007.

[43] Zehetbauer, M., Ungar, T., Kral, R., Borbely, A., Schafler, E., Ortner, B., Amenitsch, H., Bernstorff, S. (1999). Scanning X-ray diffraction peak profile analysis in deformed Cu-polycrystals by synchrotron radiation. Acta Materialia, vol. 47, no. 3, p. 1053-1061, DOI:10.1016/S1359-6454(98)00366-8. 